\title{
The IBBT w-iLab.t: a Large-scale Generic Experimentation Facility For Heterogeneous Wireless Networks
}

\author{
Stefan Bouckaert, Bart Jooris, Pieter Becue, \\ Ingrid Moerman, and Piet Demeester \\ IBBT - Ghent University, Gaston Crommenlaan 8 bus 201, 9050 Ghent, Belgium \\ stefan.bouckaert@intec.ugent.be
}

\begin{abstract}
The w-iLab.t is a large-scale generic wireless experimentation facility. Over 260 wireless nodes are installed at two different locations. Every single wireless node is equipped with multiple wireless technologies, namely IEEE 802.15.4, Wi-Fi a/b/g(/n), and on some devices also Bluetooth. Additionally, w-iLab.t provides access to software defined radio platforms and also uses them to characterize the wireless environment during an experiment. The w-iLab.t flexibility and its tools enable experimenters to design and schedule a wide range of wireless experiments, and to collect and process results in a user-friendly way.
\end{abstract}

Key words: experimentation, wireless, sensor, mesh, Wi-Fi, cognitive, testbed

\section{Hardware and tools available in w-iLab.t}

There are two operational testbed locations in w-iLab.t. The biggest installation, w-iLab.t Office, hosts wireless hardware on 200 locations spread across three floors of an office environment in Ghent, Belgium. In a more recent deployment in Zwijnaarde (close to Ghent), another 60 fixed and 20 mobile nodes are located in an unmanned utility room. A schematic overview of the Zwijnaarde architecture is provided in Figure 1. The w-iLab.t Office has a similar architecture but uses power over Ethernet in the control network. The figure shows the hardware available at every location: an embedded PC that doubles as Wi-Fi node with two wireless interfaces, (at least) one 802.15.4 sensor node, and a so-called 'environment emulator', which can be used for advanced control and monitoring of the sensor node (including power emulation, analog/digital IO, synchronized logging of results). The nodes in the Zwijnaarde testbed are additionally equipped with a Bluetooth 3.0 interface. Experimenters can choose to use any one of the available interfaces of the wireless nodes (or use of multiple interfaces simultaneously) during their experiments, in any way (e.g. for Wi-Fi: station, client, ad-hoc, custom driver,... ). In the scope of the FP7-CREW project [www.crew-project.eu], a number of cognitive radio platforms and spectrum scanners have been made available in Zwijnaarde. While in the office environment, the wireless medium 


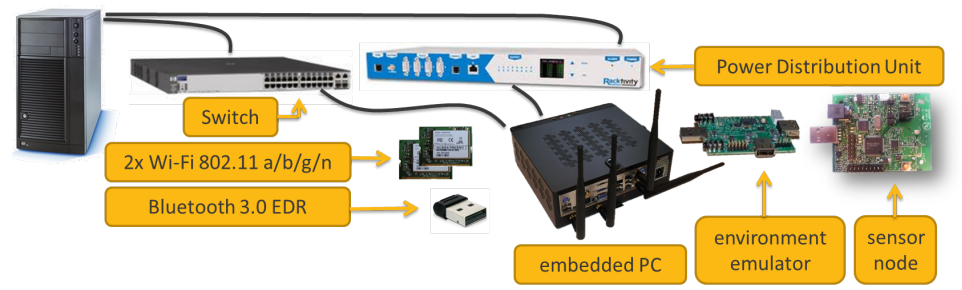

Fig. 1. Architecture of the w-iLab.t Zwijnaarde (simplified; only 1 node is shown).

is also used for day-to-day activities of the employees (wireless LAN, DECT, microwave ovens), there is very limited or no wireless activity in Zwijnaarde.

In addition to the hardware, w-iLab.t offers a set of tools to experimenters, supporting the entire experimentation lifecycle: installation of the devices (images for the embedded PC and/or firmware for the sensor nodes), configuration of the wireless nodes, scheduling experiments, event-based interaction during experiments (e.g. emulate power failure, shut down node, trigger an event on the I/O pins of the sensor node), collecting and storing of results (this is a generic system, configurable by the experimenter), and processing and visualizing results (2D graphs and/or visualization on a testbed map). The w-iLab.t Office tools are based on Motelab software [http://motelab.eecs.harvard.edu/] but were extended to support more advanced sensor node through support for the environment emulator, and to support Wi-Fi enabled experiments. The Zwijnaarde testbed tools are based on OMF [http://omf.mytestbed.net/]; OMF and the w-iLab.t OMF updates are available as open source software.

\section{Use of w-iLab.t and access info}

While it is impossible to list all possibilities of w-iLab.t in this abstract, it is important to understand that the w-iLab.t is called a generic testbed, because the configuration possibilities allow the experimenter to use the testbed for 'any' (wireless) application. As a rule of thumb: whatever experiment an experimenter would be able to execute using the testbed hardware and his/her own laptop on his/her desk, can also be executed on the w-iLab.t in an organized way. Similarly, any type of data that could be collected from a wireless networking set-up e.g. during a small-scale experiment on an experimenter's desk, can in nearly all cases also be effortlessly collected from a large-scale set-up on the w-iLab.t testbed.

The w-iLab.t testbed control interface, nodes and tools are remotely accessible over the Internet via an OpenVPN connection. Use of w-iLab.t is free of charge for non-commercial use. Commercial use is possible, but other terms may apply. For more information and to apply for an account, please consult the iLab.t homepage http://ilabt.ibbt.be, the CREW project's information portal at www.crew-project.eu/portal/wilabdoc, or contact the authors.

The research leading to these results has received funding from various national funds, and from the European Union's Seventh Framework Programme (FP7/20072013) under grant agreements $n r 258301$ (CREW) and $n r 287581$ (OpenLab). 